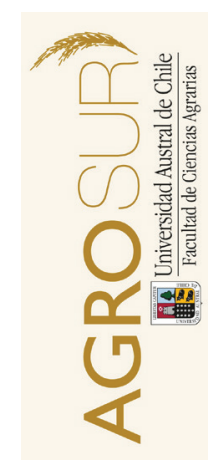

\title{
Parámetros de capacidad e intensidad del sistema poroso para evaluar la calidad física de un Andisol bajo pastoreo
}

\author{
Capacity and intensity parameters of the pore system for the evaluation \\ of the soil physical quality of an andisol under grazing
}

\author{
Dec, D. ${ }^{a, b *}$, Ivelic-Sáez, J. ${ }^{d}$, Zúñiga, F. ${ }^{a, b, c}$, Balocchi, 0. ${ }^{b, e}$, López, I. ${ }^{b, f}$, Horn, R. ${ }^{b, g}$ Dörner, J. ${ }^{a, b}$ \\ ${ }^{a}$ Instituto de Ingeniería Agraria y Suelos, Facultad de Ciencias Agrarias, \\ Universidad Austral de Chile, Casilla 567, Valdivia. \\ ${ }^{b}$ Centro de Investigación en Suelos Volcánicos, Universidad Austral de Chile. \\ ${ }^{c}$ Doctorado en Ciencias Agrarias, Escuela de Graduados, Facultad de Ciencias Agrarias, \\ Universidad Austral de Chile, Valdivia. \\ ${ }^{d}$ Instituto de Investigaciones Agropecuarias, INIA-Kampenaike. Angamos N 1056, Punta Arenas, Chile. \\ ${ }^{e}$ Instituto de Producción Animal, Facultad de Ciencias Agrarias, Universidad Austral de Chile, \\ Casilla 567, Valdivia. \\ ${ }^{f}$ Institute of Agriculture \& Environment, Collegue of Science, Massey University, New Zealand. \\ ${ }^{g}$ Institut für Pflanzenernährung und Bodenkunde, Christian Albrechts Universität zu Kiel \\ Olshausenstrasse 40, 24118 Kiel, Germany.
}

A R T I C L E I N F O

Article history:

Received 19.06.2015

Accepted 26.03.2016

Keywords:

Soil physical quality

Temporal variability of soil

properties

Volcanic soil

Grazing management

Animal trampling

Original Research Article,

Special Edition: International Year

of Soils (IYS)

Soil Science

*Corresponding author:

Dorota Dec

E-mail address:

dorota.dec@uach.cl
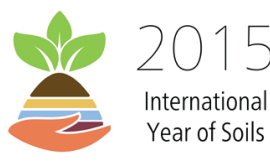

Year of Soils
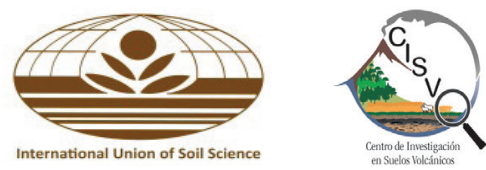

A B S T R A C T

Pastures in southern Chile are the main livestock feed throughout the year, whereas grazing events, depending on soil mechanical stability, may affect soil physical functions. In these terms, the soil physical quality is a concept, which can be determined through the response of the soil porous system functionality to the application of internal and external stresses. The purpose of this study was to analyze the effect of cattle grazing on the temporal evolution of the capacity and intensity parameters of the Andisols porous system. Six soil samplings were conducted (2-10 cm depth; the most affected by grazing), and properties such as volumetric soil water content, precompression stress (CS), air capacity (AC), air ( $\left.\mathrm{k}_{1}\right)$ and saturated hydraulic (Ks) conductivity, coefficient of pore shrinkage, and bulk density (Da) were determined. All investigated properties present temporal variations, caused by grazing events and natural wetting and drying cycles. The values defined as critical for soil compaction, have a restricted applicability in Andisols: i) while the values of bulk density are far from representing compaction problems $\left(1.7 \mathrm{~g} \mathrm{~cm}^{-3}\right)$, ii $)$ the $\mathrm{AC}(<8 \%)$ and $\mathrm{k}_{1}\left(<12 * 10^{-4}\right.$ $\mathrm{cms}^{-1}$ ) of these soils may manifest problem of soil physical quality. Finally, despite of the low Da of the Andisol, an increase in the grazing intensity during winter did not affect any investigated property negatively, highlighting the good physical quality of this soil. However, considering the properties of soils for which the critical parameters were determined, further studies are needed for Andisols to determine the range of their soil quality parameters.

\section{RESUMEN}

Las praderas en el sur de Chile constituyen la principal fuente de alimentación de ganado durante todo año. Dependiendo de la estabilidad mecánica del suelo, los eventos de pastoreo pueden afectar su funcionamiento fisico. En esos términos, la calidad física del suelo es un concepto que puede ser determinado a través de la respuesta de la funcionalidad de su sistema poroso a la aplicación de tensiones internas y externas. El propósito de este estudio fue analizar el efecto del pastoreo bovino sobre la evolución temporal de parámetros de capacidad e intensidad del sistema poroso de un Andisol. En las muestras tomadas seis veces (2-10 cm; lo más afectados por el pisoteo) se determinaron propiedades como: contenido volumétrico del agua, capacidad de soporte (CS), capacidad de aire (AC), conductividad del aire $\left(\mathrm{k}_{\mathrm{l}}\right)$ e hidráulica saturada (Ks), coeficientes de contracción de los poros y densidad aparente (Da). Todos los parámetros muestran variaciones en el tiempo, que se deben a los eventos de pastoreo y a los ciclos naturales de mojado y secado. Los valores definidos como críticos, en términos de compactación de suelos, tienen una aplicabilidad registringida en Andisoles: i) mientras los valores de Da están lejos de representar problemas 
de compactación $\left(1,7 \mathrm{~g} \mathrm{~cm}^{-3}\right)$, ii) la CA $(<8 \%) \mathrm{y} \mathrm{k}_{1}\left(<12^{*} 10^{-4} \mathrm{cms}^{-1}\right)$ pueden manifestar problemas de calidad física. Finalmente, a pesar de la baja Da del Andisol, el aumento de la intensidad de pastoreo durante el invierno no afectó negativamente ninguna propiedad investigada, indicando una buena calidad física de este suelo. Sin embargo, tomando en cuenta las propiedades de suelos para cuales fueron determinados los parámetros críticos, se recomiendan más estudios que determinen los rangos de los parámetros de calidad física de Andisoles.

Palabras clave: calidad física, variación temporal, suelo volcánico, manejo de pastoreo, pisoteo animal.

\section{INTRODUCCIÓN}

En el sur de Chile, existen cerca de 2.5 millones de hectáreas de suelos de origen volcánico que se usan para sostener recursos forrajeros (praderas y cultivos suplementarios; INE, 2007). La intensidad de uso de estos terrenos aumenta constantemente, ya que los sistemas pratenses son la principal fuente de alimento para el ganado (Balocchi, 2002). Por lo tanto, el manejo sustentable de estas superficies cobra gran importancia, y para ello la comprensión del comportamiento del suelo y su funcionamiento resulta fundamental. El comportamiento de los poros del suelo es de naturaleza dinámica debido a la acción de fuerzas externas (p. ej. producto del pisoteo animal) e internas (p. ej. ciclos de mojado y secado del suelo) que alteran la estructura del suelo y, en consecuencia, el ordenamiento espacial de sus poros (Horn y Smucker, 2005; Reszkowska et al., 2011; Dec et al., 2012; Dörner et al., 2012). Esto, es particularmente relevante para los suelos derivados de cenizas volcánicas del Centro-Sur de Chile, ya que éstos sustentan praderas que son pastoreadas por animales durante gran parte del año (Dec et al., 2011), por lo tanto, quedan expuestos a estrés de tipo mecánico e hidráulico.

El sistema poroso del suelo cumple un rol fundamental para el funcionamiento de los ecosistemas naturales e intervenidos (Blume et al., 2013), por lo tanto, su evaluación cuantitativa y cualitativa cobra gran relevancia (Dörner y Dec, 2007; Schwen et al., 2011) con el fin de valorar sus funciones. El funcionamiento de los poros del suelo depende de su volumen, distribución, continuidad y tortuosidad (Hillel, 1998). Horn y Kutilek (2009) proponen diferenciar el funcionamiento físico de los poros del suelo a través de parámetros de capacidad e intensidad. Mientras los primeros definen un estado general del suelo (p. ej. la densidad aparente y la capacidad de almacenamiento de agua en el suelo), los segundos permiten cuantificar la funcionalidad del sistema poroso (p. ej. conductividad de aire). Lo anterior es altamente relevante, ya que un suelo puede tener una gran capacidad de almacenar agua y aire (dependiendo de su profundidad y porosidad), pero estos fluidos no necesariamente serán accesibles para las plantas (dependiendo de la continuidad y tortuosidad del sistema poroso), lo cual puede verse modificado por estres mecánicos e hidráulicos (Horn y Kutilek, 2009).
Es ampliamente reconocido que los suelos derivados de cenizas volcánicas (Andisoles) presentan excelentes propiedades físicas (Ellies et al., 1993; Ellies et al., 2000; Dörner et al., 2012; Dec et al., 2012), lo que permite que estos suelos resistan altas intensidades de manejo (Balocchi, 2002). Su comportamiento físico está asociado al alto contenido de carbono orgánico (> 25\%, Soil Survey Staff, 2006) que presentan, lo que en conjunto con la presencia de minerales de corto rango de ordenamiento (p.ej. alofan), le confieren una baja densidad aparente, alta capacidad de almacenamiento y conducción de fluidos (Ellies et al., 1998; Dörner et al., 2010), como también una alta resistencia y resiliencia del sistema poroso (Dörner et al., 2011; Ivelic-Sáez et al., 2015). Además, tomando en cuenta la variación natural que presentan las propiedades fisicas de los suelos derivados de cenizas volcánicas en el tiempo (Zúñiga et al., 2015) y espacio (p. ej. Dec et al., 2012), se requieren evaluaciones exhaustivas para determinar si un incremento en la intensidad del manejo del suelo (p. ej. a través de un incremento en la carga animal o presión de pastoreo) repercute negativamente en el funcionamiento de los poros del suelo considerando valores críticos que signifiquen una degradación física del recurso. Debido a que estos cambios temporales son importantes para un manejo sustentable de los recursos pratenses, el objetivo de este trabajo fue analizar el efecto del pastoreo bovino sobre la evolución temporal de parámetros de capacidad e intensidad del sistema poroso de un Andisol, asociados a la calidad física del suelo.

\section{MATERIAL Y MÉTODOS}

\section{Sitio e historial de manejo}

El experimento se llevo a cabo en la Estación Experimental Agropecuaria Austral de la Universidad Austral de Chile (39 $48^{\circ}$ S, $73^{\circ} 15^{\prime}$ W, elevación 12 m s.n.m.). El tipo de suelo corresponde a un Duric Hapludand (Serie Valdivia; CIREN, 2003), que se caracteriza por ser moderamente profundo, con una textura franco limosa en los primeros centímetros, que se vuelve más gruesa con el aumento de la profundidad. El contenido de materia orgánica es de $16 \%$ en los primeros $10 \mathrm{~cm}$ de suelo. La topografía es compleja, presentando pendientes con gradientes de entre 5 y $8 \%$ (CIREN, 2003). El clima es templado lluvioso, con un promedio de tempera- 
tura de $12{ }^{\circ} \mathrm{C}$. Las precipitaciones anuales alcanzan los $2000 \mathrm{~mm}$, con una concentración entre mayo y agosto (Dec et al., 2011). Antes de comenzar el experimento (Abril de 2008), se sembro Ballica inglesa (Lolium perenne L.) y Trébol blanco (Trifolium repens L.). En abril de 2009, en un área total de $2400 \mathrm{~m}^{2}$ se establecieron parcelas de 20 m x 20 m en un diseño de bloques distribuidos completamente al azar. Se determinó la altura comprimida de la pradera con un plato medidor de disponibilidad de forraje (Jenquip Filip's Manual Folding Plate Meter). Se utilizó como criterio de ingreso de pastoreo la disponibilidad de forraje (1700-2400 kgMS $\left.\mathrm{ha}^{-1}\right)$, mientras que el criterio de salida correspondió a un rango de disponibilidad entre 1200-1500 kgMS ha-1 (MS = materia seca). Durante la primera parte de la investigación, el pastoreo se realizó con una carga animal instantánea de 250 vacas ha-1 día $^{-1}$ en todas las parcelas. A partir de junio de 2009 se realizaron pastoreos de invierno con dos cargas animales instantáneas: la primera con 200 vacas ha ${ }^{-1}$ día $^{-1}$ (parcelas A) y la segunda con 50 vacas ha ${ }^{-1}$ día $^{-1}$ (parcelas B). Las vacas usadas en el experimento corresponden a Frisón Negro en lactancia de aproximadamente $500 \mathrm{~kg}$ de peso vivo. La alta carga enimal elegida fue calculada tomando en cuenta directrices de Abarzua et al. (2007).

\section{Muestreo de suelos}

Durante el periodo investigado, se llevaron a cabo seis muestreos del suelo (M) en las siguientes fechas: M1: 15.04, M2: 20.04, M3: 28.05, M4: 22.06, M5: 26.06, M6: 30.08 todas realizadas el año 2009. Las fechas fueron definidas de acuerdo con los pastoreos (definidos según la disponibilidad de MS) y los ciclos de mojado y secado, tomando como referencia los eventos de lluvia y el contenido volumétrico de agua del suelo. Los muestreos M1 - M4 se llevaron a cabo bajo condiciones de pastoreo con una carga instantánea de 250 vacas ha ${ }^{-1}$, mientras que los muestreos M5 - M6 se realizaron durante el pastoreo de invierno (200 y 50 vacas ha-1 día $^{-1}$ ). El muestreo M5 se llevó a cabo directamente después del pastoreo de invierno.

En las fechas indicadas se recolectaron muestras no disturbadas de suelo $(n=7)$ entre 2 y $10 \mathrm{~cm}$ de profundidad en cilindros metálicos de $230 \mathrm{~cm}^{3}$. Con estas muestras de suelo se determinó la curva de retención de agua, la conductividad de aire $\left(\mathrm{k}_{\mathrm{l}}\right)$ y la curva de contracción del suelo. Adicionalmente, para determinar la capacidad de soporte del suelo (CS), por bloque se recolectaron 6 muestreas en cilindros metálicos (volumen de $120 \mathrm{~cm}^{3}$ ) para someterlos a ensayo de consolidación.

\section{Determinación de la calidad física del suelo}

El análisis de la calidad física del suelo se realizó por medio de la determinación de parámetros de ca- pacidad (capacidad de aire, índices de contracción del suelo y capacidad de soporte) e intensidad (conductividad de aire e hidráulica saturada) del sistema poroso (Horn y Kutilek, 2009).

Antes de comenzar el análisis de laboratorio, las muestras fueron lentamente saturadas por ascenso capilar de agua por varios días. Una vez alcanzada la saturación, las muestras fueron sometidas a tensiones de agua crecientes de: 1, 2, 3, 6 (en bandejas de arena), 15, 30 y 50 (en cámaras de presión) $\mathrm{kPa}$. Cuando las muestras alcanzaron un equilibrio con la tensión aplicada, se determinó su peso y deformación vertical. Las muestras fueron pesadas en una balanza de precisión (Precisa, 0,01 g de precisión). La deformación vertical o contracción del suelo fue medida en 7 puntos de cada muestra por medio de un profundímetro (exactitud $0,05 \mathrm{~mm}$ ). Finalmente, las muestras fueron secadas a $105^{\circ} \mathrm{C}$ por 24 horas para determinar su densidad aparente (Sandoval et al., 2012).

A partir de la deformación vertical del suelo se calcularon los coeficientes de contracción: COLE (coeficiente de extensabilidad lineal) y PSI (coeficiente de contracción de los poros (Grossman et al., 1968; Peng et al., 2007, 2009). Para ello se utilizaron las siguentes ecuaciones:

$$
C O L E=\frac{L_{a}-L_{b}}{L_{105^{\circ} \mathrm{C}}}
$$

Ec. 1

Donde:

$L_{a}=$ Longitud de la muestra saturada [cm].

$L_{b}=$ Longitud de la muestra a una tensión determinada [cm].

$L_{105^{\circ} \mathrm{C}}=$ Longitud de la muestra después de secado a una temperatura de $105^{\circ} \mathrm{C}[\mathrm{cm}]$.

$$
P S I=\frac{\Delta V_{t}}{\Delta V_{p}}
$$

Donde:

$\Delta V_{t}=$ Volumen de contracción del suelo [cm].

$\Delta V_{p}=$ Volumen de pérdida de agua [cm].

Adicionalmente, cuando las muestras alcanzaron una tensión de agua de $6 \mathrm{kPa}$, se determinó la capacidad (CA) y conductividad de aire $\left(\mathrm{k}_{\mathrm{l}}\right)$. La capacidad de aire fue calculada como la diferencia entre el contenido del agua en saturación y drenadas a $6 \mathrm{kPa}$ de tensión, considerando la contracción que sufrió el suelo durante el secado (Dörner et al., 2010). La conductividad del aire se midió 3 veces por muestra mediante de un flujométro de aire, en donde, luego de aplicar una diferencia de presión constante y menor a $1 \mathrm{hPa}$, se inicia un flujo laminar ascendente a través de la muestra de suelo. Este flujo es registrado en un medidor de flujo de aire (Key Instruments, Trevore, USA), en un rango de conductividad de aire entre 0,1 a $10 \mathrm{~L} \mathrm{~min}^{-1}$ (Dörner y Horn, 2006). 
La conductividad hidráulica saturada se (Ks) determinó en muestras no disturbadas de sueloque fueron saturadas por capilaridad, posteriormente pesadas y colocadas en permeámetro de agua (Eijkelkamp, modelo 09.02.01.25). Ks fue medida en 48 horas despues de iniciar el flujo del agua através de la muestra del suelo (Dörner et al., 2010).

La curva de consolidación se determinó en muestras no disturbadas de suelo $(\mathrm{n}=6)$, equilibradas a una tensión de $6 \mathrm{kPa}$, mediante el uso de un odómetro (Controls T303). Se realizó un ciclo de cargas estáticas sucesivas $(6.25,12.5,25,50100200$ y $400 \mathrm{kPa})$ y un ciclo de descarga $(200,100,50,6.25$ y $1 \mathrm{kPa})$ cada 6' para ambos ciclos, durante los cuales se registró la deformación vertical del suelo (0,001 mm de precisión). A partir de la curva de consolidación y usando el método matemático propuesto por Baumgartl y Köck (2004), que se basa en el método gráfico de Casagrande (1936), se determinó la capacidad de soporte del suelo (CS), utilizando el software RETC v 6.02 (2005-2009).

La conductividad hidráulica saturada se determinó mediante el uso de un permeámetro de carga constante según la metodología descrita por Hartge y Horn (2009).

\section{Análisis estadístico}

Se determinaron los valores promedio con sus respectivos errores estándar. Para normalizar los valores de conductividad de aire y conductividad hidráulica saturada, estos fueron transformados a logaritmo de acuerdo a lo sugerido por Hartge y Horn (2009).

\section{RESULTADOS Y DISCUSIÓN}

\section{Análisis del efecto del pastoreo sobre los parámetros de capacidad del sistema poroso del suelo}

El contenido volumétrico de agua del suelo durante el periodo de estudio varió entre valores cercanos al punto de marchitez permanente (14\%) y capacidad de campo (40\%) (Cuadro 1, ver Dec et al., 2012). Los cambios en el contenido de agua son relevantes ya que: i) condicionan la estabilidad mecánica del suelo (Ellies, 1988; Osunbitan et al., 2005; Dec et al., 2011; Dorner et al., 2012), por lo tanto, la respuesta del suelo frente al pisoteo de los animales, y ii) contribuyen a la dinámica estructural de los suelos debido a la formación de meniscos de agua que permiten la contracción, formación de grietas y, por tanto, estructuración de los suelos (Dörner et al., 2011).

En la evolución de la porosidad de suelo (Cuadro 1), no se observaron diferencias entre los tratamientos y muestreos, ya que esta propiedad depende del valor de la densidad aparente (Da), que no presentó variaciones en el periodo investigado. Los valores de Da determi- nados en este estudio son característicos para un suelo volcánico (<0,9 $\mathrm{Mg} \mathrm{m}^{-3}$, WRB, 2014) y se encuentran lejos de los típicos de suelos minerales que presentan problemas de compactación (p.ej. Da $\geq 1,7 \mathrm{Mg} \mathrm{m}^{-3}$; Horn y Fleige, 2009). Sin embargo, esto no quiere decir que estos suelos no puedan presentar problemas de compactación. Dörner et al. (2013a), indican que en la medida que la Da de los suelos derivados de cenizas volcánicas se acerque a $0,9 \mathrm{Mg} \mathrm{m}^{-3}$ pueden alcanzar valores de capacidad y conductividad de aire restrictivos para un adecuado intercambio gaseoso.

La capacidad de aire (CA) del suelo presenta una evolución temporal asociada a eventos de estrés mecánicos e hidráulicos (Figura 1). Este parámetro es uno de los más susceptibles a los cambios en el manejo del suelo (Dörner et al., 2011; Reszkowska et al., 2011; Dec et al., 2012), ya que al ser sensible al efecto del estrés mecánico (Gebhardt et al., 2009) es modificado en primera instancia (Richard et al., 2001; Fujikawa y Miyazaki, 2005). Los niveles de CA observados variaron entre un 8 y 14\%, alcanzándose el valor de crítico de 8\% (en M6), señalado como indicador de problemas asociados a la compactación del suelo por Horn y Fleige (2009), lo que puede relacionarse con el pastoreo bajo condiciones de mayor contenido de agua en el suelo. Los valores de CA obtenidos son característicos para suelos de origen volcánico (Armas-Espinel et al., 2003; Dörner et al., 2009b, 2010). En ese contexto y de acuerdo a lo observado en otros suelos de origen volcánico (p. ej. Dörner et al., 2011) el Andisol fue capaz de resistir la deformación provocada por los animales en pastoreo (excepto en M6), lo que sumado a su alta capacidad de resiliencia (Dörner et al., 2009a, 2011), permite que estos suelos soporten altas intensidades de manejo (Dec et al., 2012); sin embargo, existe una variación en el tiempo que debe ser considerada en la evaluación de la calidad física del suelo.

Una de las maneras para comprender el funcionamiento del suelo es a través del estudio de su estabilidad mécanica, ya que permite establecer el comportamiento de los poros del suelo frente a un evento de estrés mecánico (Horn y Fleige, 2009), lo que puede ser determinado por medio de la capacidad de soporte (CS, Figura 2). Esta propiedad del suelo varía en el tiempo, presentando valores entre 26 y $55 \mathrm{kPa}$. Para los tres primeros muestreos (M1-M3), CS aumenta como resultado de la presión generada en los eventos de pastoreo (Reszkowska et al., 2011) que se llevaron a cabo entre M1 y M2. A pesar de incremento en la CS, ésta no alcanza los valores críticos que indican problemas de compactación de suelo (CS $>90 \mathrm{kPa}$; Horn y Fleige, 2009). Sin embargo, despues del muestreo M3 la CS disminuye, lo que evidencia que el suelo fue capaz de soportar la carga animal con una humedad entre 40 y $50 \%$ de saturación de agua en los poros, (Cuadro 1) durante la estación de invierno (M5 y M6), indicando 
Cuadro 1: Características generales del suelo.

Table 1: General characteristics of the studied soil.

\begin{tabular}{cccccc}
\hline Fecha & Muestreo & $\begin{array}{c}\theta_{c} \\
{[\text { Vol. } \%]}\end{array}$ & $\begin{array}{c}\text { PT } \\
{[\%]}\end{array}$ & $\begin{array}{c}\theta_{S} \\
{[\%]}\end{array}$ & $\begin{array}{c}\text { Dap } \\
{\left[\mathrm{Mg} \mathrm{m}^{-3}\right]}\end{array}$ \\
\hline 15.04 .2009 & M1 & $17 \pm 0,3$ & 0,69 & 0,24 & $0,71 \pm 0,01$ \\
20.04 .2009 & M2 & $15 \pm 0,4$ & 0,71 & 0,22 & $0,67 \pm 0,01$ \\
28.05 .2009 & M3 & $28 \pm 0,3$ & 0,69 & 0,41 & $0,71 \pm 0,01$ \\
22.06 .2009 & M4 & $31 \pm 0,6$ & 0,69 & 0,45 & $0,72 \pm 0,01$ \\
26.06 .2009 & M5A & $29 \pm 0,3$ & 0,70 & 0,41 & $0,69 \pm 0,01$ \\
30.08 .2009 & M5B & $27 \pm 0,7$ & 0,70 & 0,39 & $0,70 \pm 0,01$ \\
& M6A & $35 \pm 0,1$ & 0,69 & 0,50 & $0,72 \pm 0,01$ \\
& M6B & $34 \pm 0,1$ & 0,69 & 0,50 & $0,72 \pm 0,01$ \\
\hline
\end{tabular}

$\theta_{\mathrm{c}}$ y $\theta_{\mathrm{s}}=$ Contenido volumétrico de agua y saturación de agua en los poros durante el muestreo de suelo; PT = Porosidad total; Dap: Densidad aparente. Se presentan valores promedio \pm error estándar.

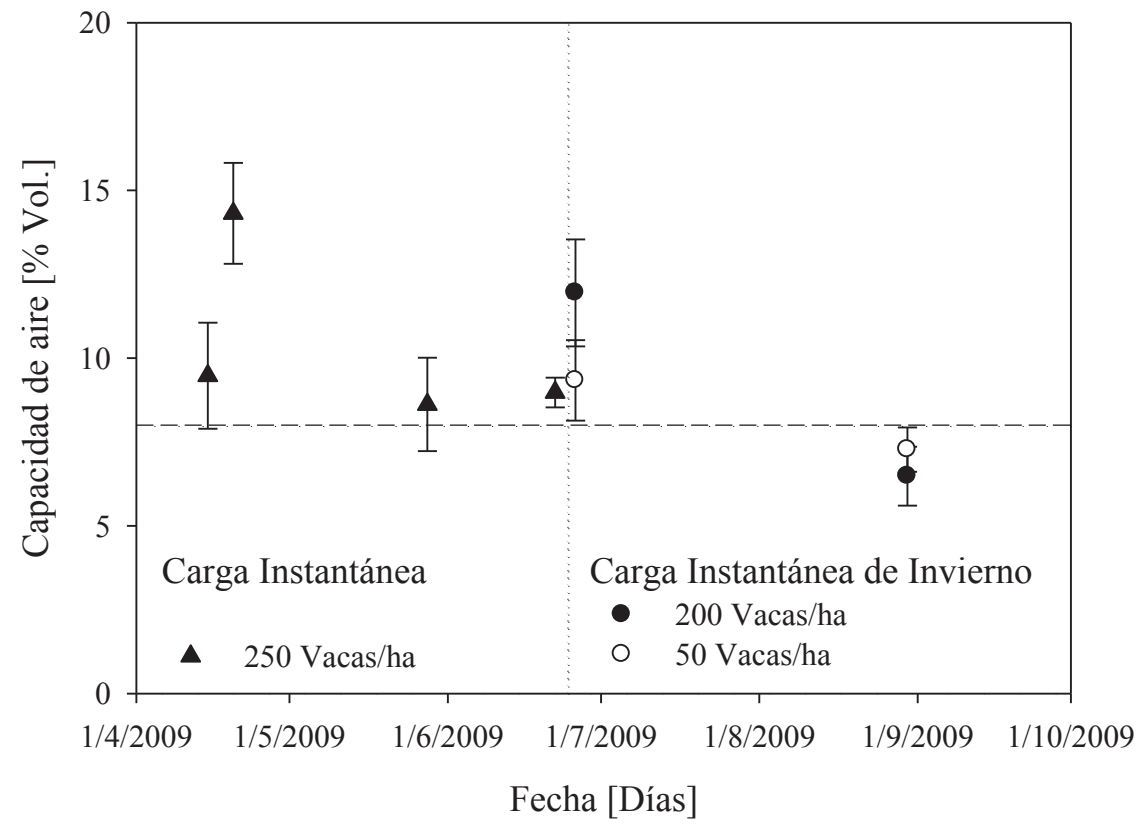

Figura 1. Capacidad del aire (AC) en función del tiempo y bajo el efecto de los distintos pastoreos. Las barras indican \pm 1 error estándar (n=7). La línea entrecortada paralela al eje x muestra el valor crítico de referencia de problemas de compactación de acuerdo a Horn y Fleige (2009).

Figure 1. Air capacity (AC) during the sampling period under different grazing intensities. Bars indicate \pm 1 standard error $(\mathrm{n}=7)$. Dashed lines parallel to $\mathrm{x}$-axis indicate reference critical values according to Horn and Fleige (2009).

una gran estabilidad estructural que permite mantener las funciones del sistema poroso del suelo (Dec et al., 2012). Esto se debe a la reacción de este tipo de suelos a los ciclos de mojado y secado (especialmente visible entre M2 y M3, como consecuencia de varios ciclos de humectación y secado entre muestreos) y el crecimiento de las raíces creando nuevos poros, los que permiten la recuperación del sistema poroso del suelo (Czarnes et al., 2000; Horn y Smucker, 2005). Estos procesos mo- difican la distribución de espacio poroso (Figura 1) y las funciones relacionadas (Dörner et al., 2011).

En cuanto a la intensidad de pastoreo, no se observó un efecto claro para la CA (Figura 1) y CS (Figuras 1 y 2 , respectivamente) del suelo producto del aumento en la carga animal (de 50 a 200 vacas ha-1 día ${ }^{-1}$ ). Esto indica que bajo las condiciones del estudio el suelo logró soportar el estrés mecánico y luego recuperarse de los eventos de pastoreo, siendo este comportamiento con- 


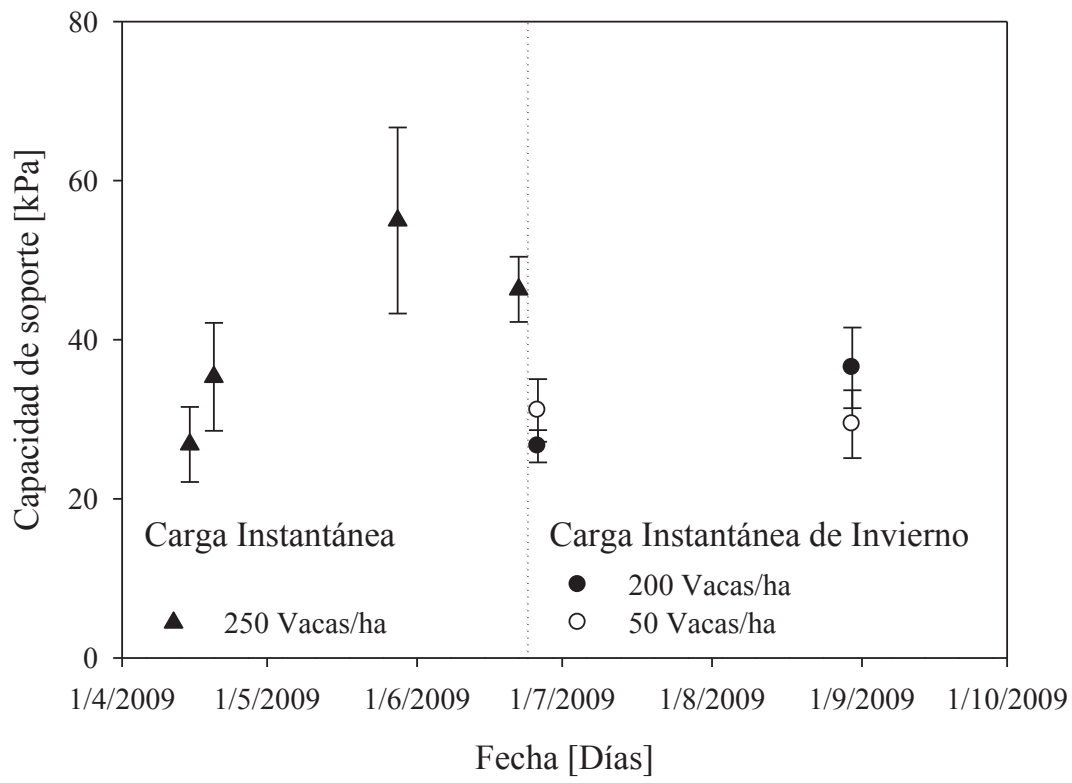

Figura 2. Capacidad de soporte (CS) en función del tiempo y bajo el efecto de los distintos pastoreos. Las barras indican \pm 1error estándar ( $\mathrm{n}=7)$.

Figure 2. Precompression stress (CS) during the sampling period under different grazing intensities. Bars indicate \pm 1 standard error $(n=7)$.

sistente con la resistencia y resiliencia de los suelos derivados de ceniza volcánicas (Ivelic-Sáez et al., 2015), debido a que el alto contenido de materia orgánica que presentan (16\% para esta serie) les confiere una gran elasticidad (Ellies, 1988; Dörner et al., 2011).

La dinámica estructural en suelos bajo pastoreo esta dada por ciclos de carga y descarga (p.ej. pisoteo animal en Rezkowska et al., 2011) y ciclos de mojado y secado (Dec et al., 2012). En ese contexto, el fenómeno de la contracción en estos suelos depende del manejo animal y del clima (Peng et al., 2009) lo que permite, en conjunto con la actividad biológica del suelo (Chenu y Cosentino, 2011), una recuperación de sus funciones debido a la creación de poros mas continuos (Dörner et al., 2011; Zúñiga et al., 2015). Los valores de COLE y PSI del presente estudio (Figura 3 y 4) están en los rangos esperados para los suelos de origen volcánico (Dörner et al., 2009b), los que se caracterizan por poseer una gran capacidad de contracción (COLE $>0,06$, Peng et al., 2007). El efecto del pisoteo con una carga animal constante (M1-M4), pero con contenidos de agua de suelo que permiten sostener la carga animal, se puede apreciar mediante una ligera disminución de COLE y PSI a través del tiempo. El aumento en el contenido volumétrico del agua del suelo (M4 - M6) produce que el pisoteo tenga un efecto destructivo en la estructura, provocando un incremento de los poros medios y finos del suelo de tal manera que aumenta la capacidad de contracción de los suelos (Peng et al., 2007).

\section{Análisis del efecto del pastoreo sobre los parámetros de intensidad del sistema poroso del suelo}

Según el Schoenholtz et al. (2000), el concepto de la calidad del suelo incluye la evaluación de sus propiedades y los procesos que permiten analizar la capacidad de éste para funcionar eficazmente como un componente del ecosistema. Por lo mismo, la definición de calidad y la elección de los parámetros que la determinan dependen de la finalidad que se persigue investigando un ecosistema. La presente investigación se llevó a cabo en una pradera establecida sobre un suelo derivado de cenizas volcánicas, el que, debido a sus propiedades y manejo, presenta variaciones de sus características en el tiempo (Figura 1 - 6). Sin embargo, el análisis del sistema poroso requiere conocer el funcionamiento de los poros del suelo. Esta información se obtiene determinando parámetros como la conductividad de aire y conductividad hidráulica, que se caracterizan por ser muy sensibles a los cambios en la estructura del suelo, ya que reflejan el volumen de macroporos y su nivel de organización (Dörner et al., 2010).

El concepto de calidad del suelo debe reflejar su funcionalidad (Carter et al., 1997), lo que dependerá no solo del volumen de poros a una tensión determinada, sino que también de la habilidad que éstos tengan para conducir el agua o aire, la que según varios autores puede ser negativamente afectada debido al pastoreo animal (Martínez y Zinck, 2004; Peth y Horn, 2006; 


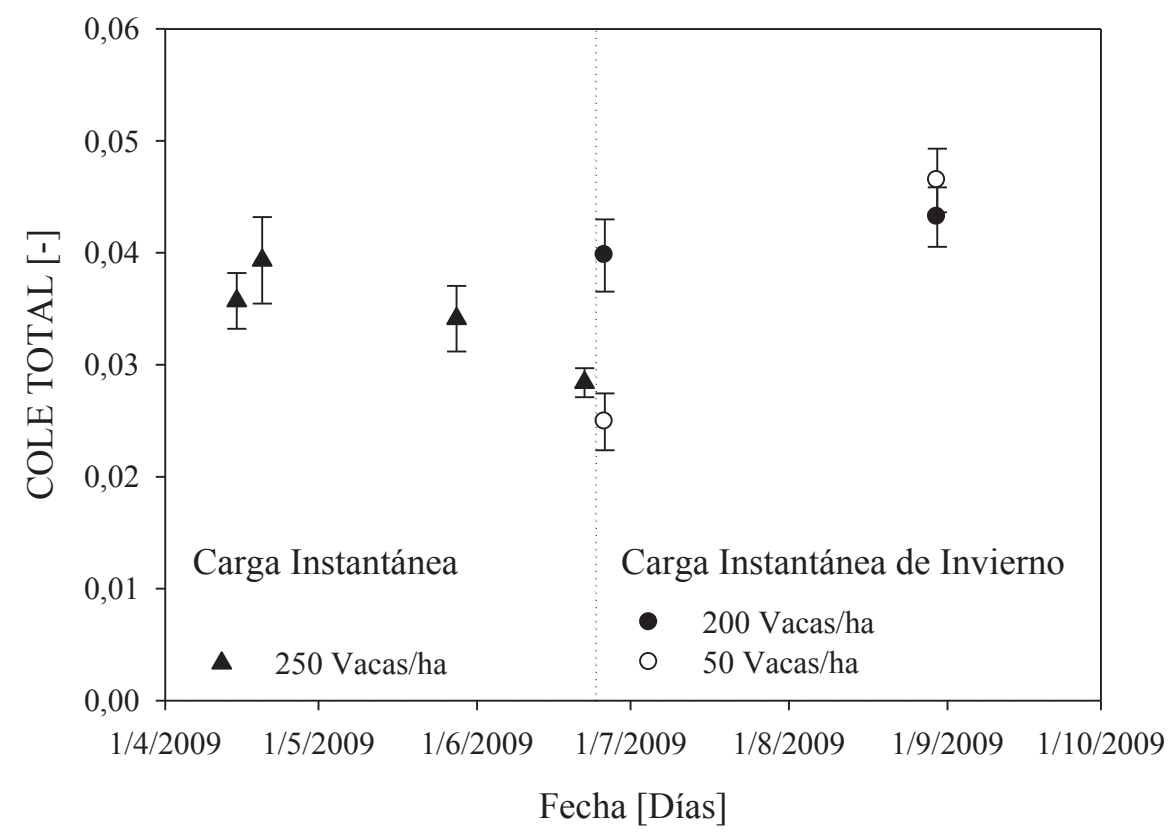

Figura 3. Coeficiente de Extensibilidad Lineal (COLE) en función del tiempo y bajo el efecto de los distintos pastoreos. Las barras indican \pm 1 error estándar $(n=7)$.

Figure 3. Coeficient of Lineal Extensibility (COLE) during the sampling period under different grazing intensities. Bars indicate \pm 1 standard error $(n=7)$.

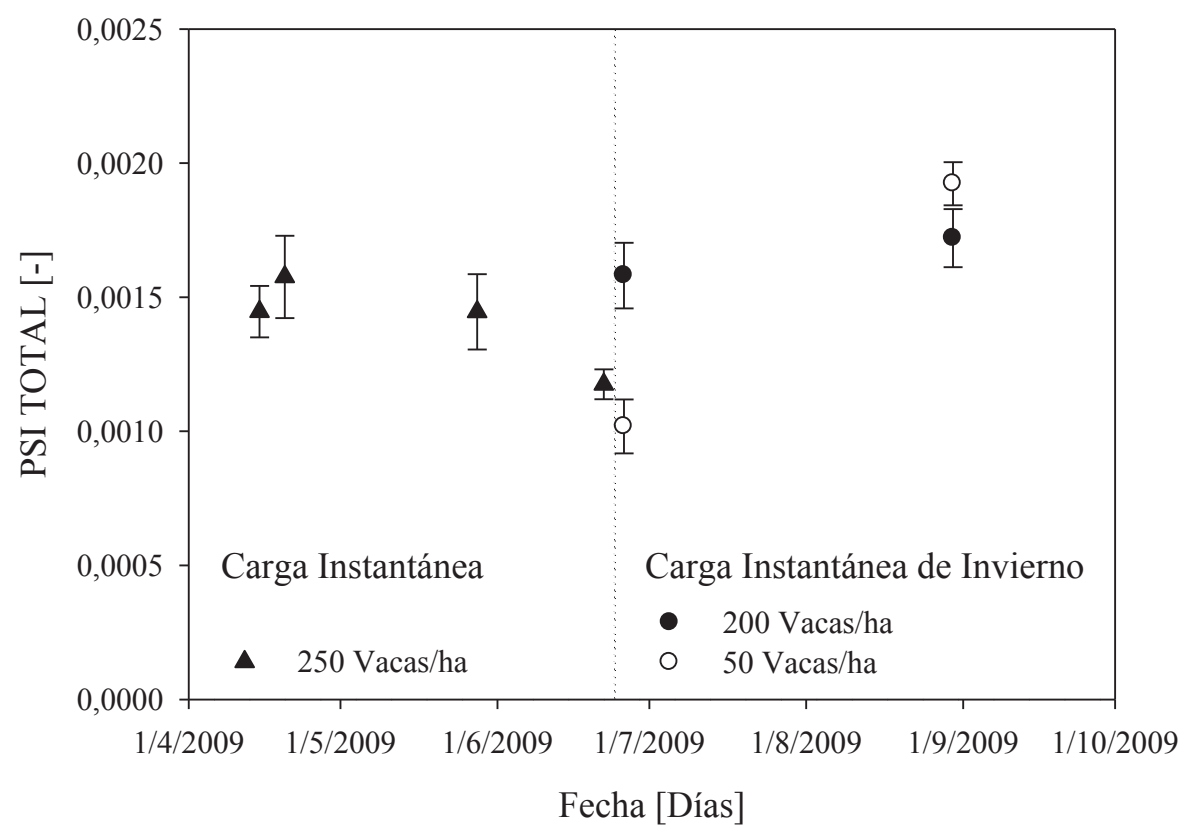

Figura 4. Indice de Contracción de los Poros (PSI) en función del tiempo y bajo el efecto de los distintos pastoreos. Las barras indican \pm 1error estándar $(\mathrm{n}=7)$.

Figure 4. Pore Shrinkage Index (PSI) during the sampling period under different grazing intensities. Bars indicate \pm 1 standard error $(n=7)$. 


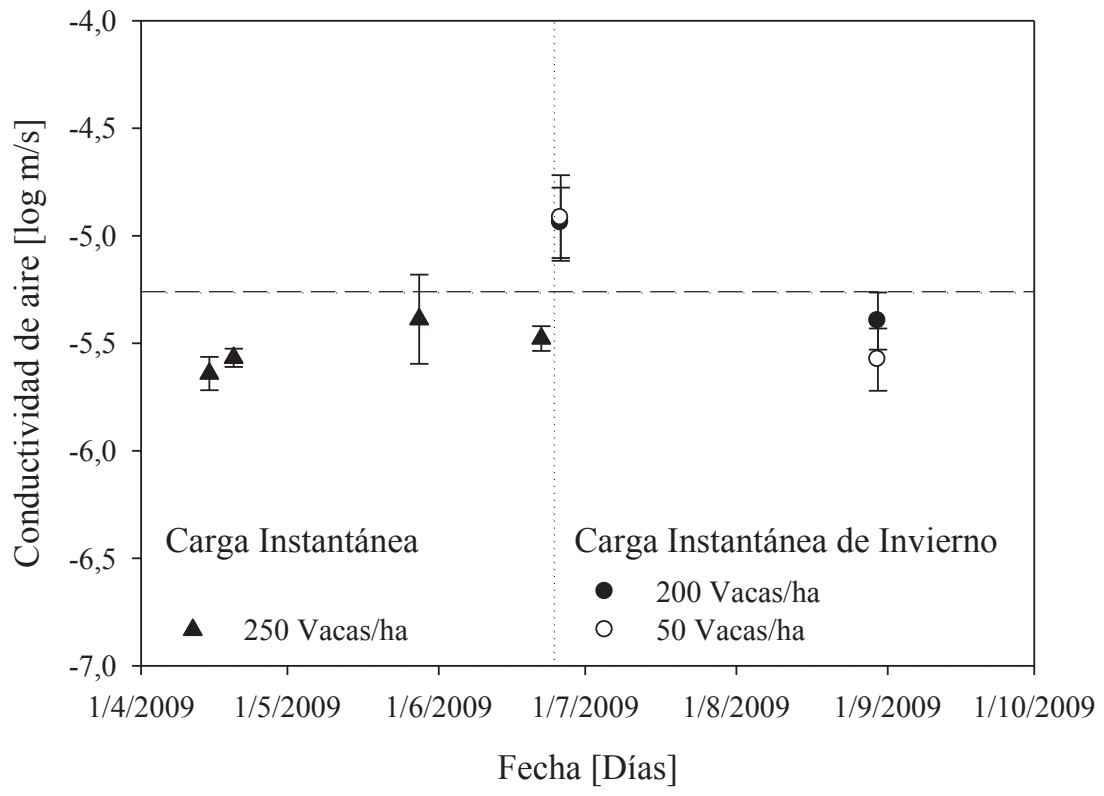

Figura 5. Conductividad del aire $\left(\mathrm{k}_{\mathrm{l}}\right)$ en función del tiempo y bajo el efecto de los distintos pastoreos. Las barras indican \pm 1 error estándar $(n=7)$. La línea entrecortada paralela al eje x muestra el valor crítico de referencia de problemas de compactación de acuerdo a Horn y Fleige (2009).

Figure 5. Air conductivity $\left(\mathrm{k}_{\mathrm{l}}\right)$ during the sampling period under different grazing intensities. Bars indicate \pm 1 standard error $(\mathrm{n}=7)$. Dashed lines parallel to $\mathrm{x}$-axis indicate reference critical values according to Horn and Fleige (2009).

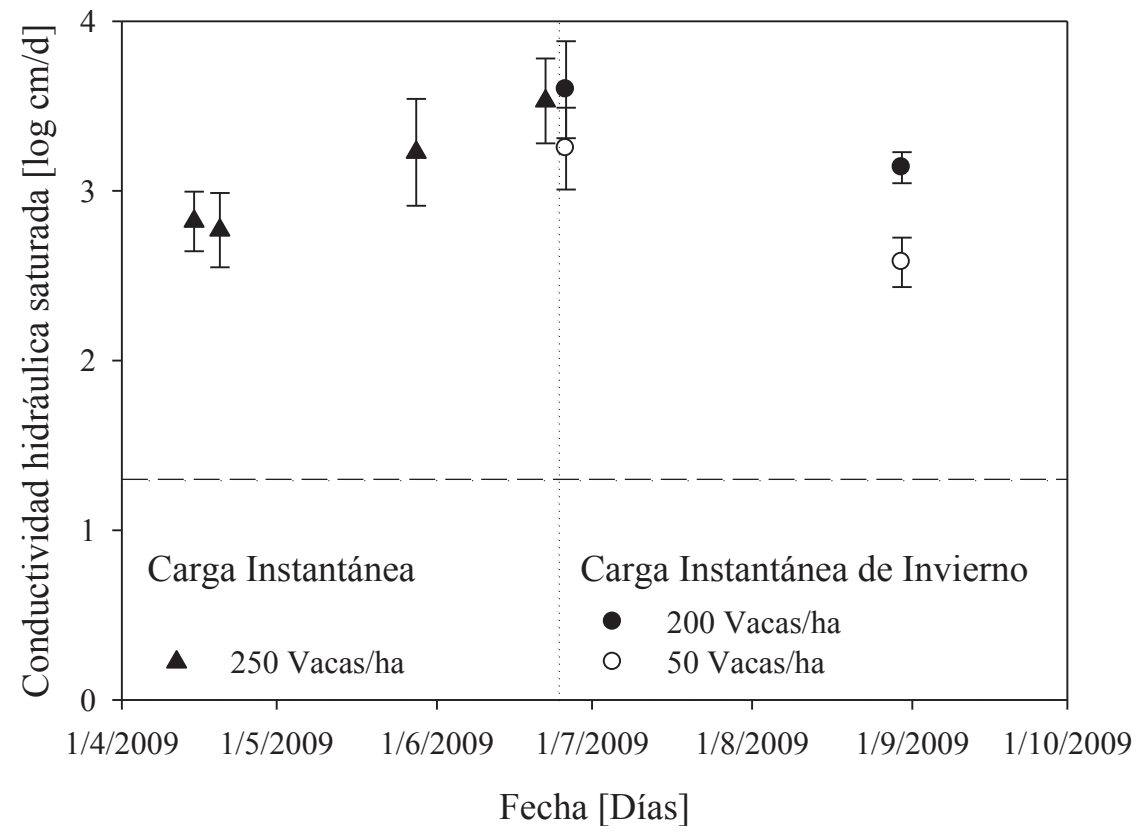

Figura 6. Conductividad hidraulica saturada (Ks) en función del tiempo y bajo el efecto de los distintos pastoreos. Las barras indican \pm 1 error estándar $(\mathrm{n}=10)$. La línea entrecortada paralela al eje x muestra el valor crítico de referencia de problemas de compactación de acuerdo a Horn y Fleige (2009).

Figure 6. Coeficient of Lineal Extensibility (COLE) during the sampling period under different grazing intensities. Bars indicate \pm 1 standard error $(n=10)$. Dashed lines parallel to $x$-axis indicate reference critical values according to Horn and Fleige (2009). 
Reszkowska et al., 2011;). Las variaciones temporales observadas para conductividad de aire (Figura 5) y la conductividad hidráulica saturada (Figura 6) fueron menos pronunciadas en comparación a los otros parámetros determinados. La conductividad de aire $\left(\mathrm{k}_{\mathrm{l}}\right)$ alcanza valores iguales o menores al valor crítico $(-5,2 \log$ $\mathrm{m} \mathrm{s}^{-1}$ ) que indica problemas de intercambio gaseoso por convección. Durante el periodo investigado se llevaron a cabo dos pastoreos: libre (entre M1 y M2) y con distintas intensidades (entre M5 y M6), los que afectaron el flujo de aire por convección en el suelo. Para el M5, ejecutado en invierno, inmediatamente después del pastoreo con distinta carga animal y bajo un alto contenido de agua (Cuadro 1), la conductividad de aire aumentó por sobre el valor crítico (Horn y Fleige, 2009), lo que se asocia a un ligero incremento en la capacidad de aire. Sin embargo, en M6 se puede notar que producto del pastoreo $\mathrm{k}_{1}$ vuelve a valores menores al descrito en la literatura como crítico. Durante este evento las pezuñas de los animales deforman la superficie del suelo, provocando un reordenamiento de los agregados y una reducción de los macroporos, que son responsables del transporte de aire (Richard et al., 2001; Fujikawa y Miyazaki, 2005). En el caso de la conductividad hidráulica saturada (Figura 6), para los primeros cuatro muestreos, se observó un aumento de esta propiedad, mientras que despues del pastoreo de invierno (entre M4 y M5) Ks tiende a disminuir. El valor mínimo de Ks alcanzado fue de 2,5 log cm día ${ }^{-1}$, el que no afecta el funcionamiento del sistema poroso y tampoco el crecimiento de las raíces de las plantas, debido a que se aleja del rango considerado crítico $\left(1-1,3 \log \mathrm{cm}\right.$ día $\left.^{-1}\right)$ por Horn y Fleige (2009). No se observó un efecto negativo del incremento de la carga animal sobre la Ks. Esto demuestra que las cargas de 50 y 200 vacas ha ${ }^{-1}$ día $^{-1}$ no afectaron la capacidad de conducción del agua del Andisol estudiado, respaldando nuevamente la hipótesis de que este tipo de suelos presenta una calidad física que difiere de los niveles críticos propuestos en la literatura, tal como lo presentan y discuten Dörner et al. (2013b). Estas propiedades del suelo y la permanencia en el tiempo de las características de este sistema (continuidad, interconexión y tortuosidad; Moldrup et al., 2001), su resistencia y resiliencia (Dorner et al., 2011; Dec et al., 2012), se deben no solo a los propiedades ándicas del suelo evaluado (Baumgarten et al., 2013), sino que también se deberían relacionar estrechamente a la actividad microbiológica del suelo (Oades, 1993), de lo que se requiere hacer más investigación (Zúñiga et al., 2015). El conjunto de estas propiedades y dependencias permite que el suelo manifieste respuestas rápidas y positivas a los ciclos de mojado y secado (cambios en contenido de agua; Cuadro 1) lo que favorece el buen funcionamiento de los poros del suelo y por consecuencia mayores niveles en los parámetros que influyen en manera positiva la calidad física del Andisol evaluado.

\section{CONCLUSIONES}

Todos los parametros investigados muestran variaciones en el tiempo, que se deben a los eventos de pastoreo y a los ciclos naturales de mojado y secado, reflejando la respuesta del suelo a los cambios en su contenido de agua y su efecto en la resistencia mecánica y agregación.

Estos cambios influyen de manera positiva los parámetros de intensidad y capacidad del sistema poroso, aumentando su estabilidad estructural y resistencia, lo que se vio reflejado especialmente en el pastoreo realizado bajo alto contenido de humedad, cuando el suelo fue capaz soportar una alta carga animal (capacidad de soporte), sin afectar significativamente la capacidad del aire, como tampoco la funcionalidad de sistema poroso (conductividad del aire y conductividad hidráulica saturada).

Los valores definidos como críticos, en términos de compactación de suelos, tienen una aplicabilidad restringida en Andisoles. Mientras los valores de densidad aparente están lejos de representar problemas de compactación, la conductividad de aire de estos suelos, es muy sensible a los cambios en la estructura del suelo, bordeando valores críticos que reflejan problemas de calidad física de suelos. Tomando en cuenta, que los valores críticos corresponden a suelos con otro origen, es necesario realizar más investigación para respaldar la información obtenida.

Para todos los parámetros del sistema poroso no se observó un efecto negativo producto del aumento de intensidad de pastoreo (de 50 a 200 vacas ha $^{-1}$ día $^{-1}$ ) lo que se debe a una gran estabilidad del Andisol estudiado e indica su buena calidad física. Sin embargo, esto no implica que estos suelos puedan soportar altas cargas de manera sostenida, sino que más bien es una alerta para conservar la calidad de sus funciones.

\section{AGRADECIMIENTOS}

Este trabajo fue llevado a cabo gracias al financiamiento del proyecto FONDECYT 3080039 y Proyecto de “Consorcio Lechero" Project M2P12 (4101.39.14).

\section{REFERENCIAS}

Abarzúa, A., Anwandter, V., Balocchi, O., Canseco, C., Demanet, R., Lopetegui, J., Parga, J., Teuber, N., 2007. Manejo del pastoreo. Imprenta America. ISBN No 978-956-310909-2.

Armas-Espinel, S., Hernández-Moreno, J., Muñoz-Carpena, R., Regalado, C., 2003. Physical properties of "sorriba"- cultivated volcanic soils from Tenerife in relation to andic diagnostic parameters. Geoderma 117, 297-311.

Balocchi, O., 2002. Praderas y recursos forrajeros en la zona sur de Chile,in: Amtman, C., Mujica, F., Vera, B. (Eds.), Pequeña agricultura en la Región de Los Lagos, Chile. 
Ediciones de la Universidad Austral de Chile, Valdivia, Chile. pp. 59-73.

Baumgarten, W., Dörner, J., Horn, R., 2013. Microstructural development in volcanic ash soils from South Chile. Soil and Tillage Research 129, 48-60.

Baumgartl, Th., Köck, B., 2004. Modeling volume change and mechanical properties with hydraulic models. Soil Science Society American Journal 68, 57-65.

Blume, H.-P., Brümmer, G.W., Horn, R., Kandeler, E., Kögel-Knabner, I., Kretzschmar, R., Stahr, K., Wilke, B.-M., 2013. Scheffer y Schachtschabel: Lehrbuch der Bodenkunde. Spektrum Akademischer Verlag, Heidelberg. ISBN 978-3-8274-2251-4.

Carter, M.R., Gregorich, D.W., Anderson, J.W., Doran, H.H., Janzen, F.J., Pierce. 1997. Chapter 1 Concepts of soil quality and their significance, in: Gregorich, E.G., Carter, M.R., (Eds.), Developments in Soil Science. Soil Quality for Crop Production and Ecosystem Health Volume 25, pp. 1-19.

Casagrande, A., 1936. Characteristics of cohesive soils affecting the stability of slopes and Earth fill. Journal of the Boston Society of Civil Engineers 23, 13-32.

Chenu, C., Cosentino, D., 2011. Microbial regulation of soil structural dynamics, in: Ritz, K., Young, I. (Eds.), The Architecture and Biology of Soils: Life in Inner Space. pp. 37-70, http://dx.doi.org/10.1079/9781845935320.00 37.

Centro de Información de Recursos Naturales (CIREN). 2003. Estudio agrológico X Región. Tomo 2. 412 p. ISBN 9567153-49-3. Centro de Información de Recursos Naturales, Santiago, Chile.

Czarnes, S., Hallett, P.D., Bengough, A.G., Young, I.M., 2000. Root- and microbial-derived mucilages affect soil structure and water transport. European Journal of Soil Science 51, 435-443.

Dec, D., Dörner, J., Balocchi, O., 2011. Temporal and spatial variability of structurE dependent properties of a volcanic ash soil under pasture in southern Chile. Chilean Journal of Agricultural Research 71, 293-303.

Dec, D., Dörner, J., Balocchi, O., López, I., 2012. Temporal dynamics of hydraulic and mechanical properties of an Andosol under grazing. Soil and Tillage Reseacrh 125, 44-51.

Dörner, J., Horn, R., 2006. Anisotropy of pore functions in structure Stagnic Luvisols in the Weichselien moraine region in N Germany. Journal of Plant Nutrition and Soil Science 169, 213-220.

Dörner, J., Dec, D., 2007. La permeabilidad del aire y conductividad hidráulica saturada como herramienta para la caracterización funcional de los poros del suelo. Revista de la Ciencia del Suelo y Nutrición Vegetal 7, 1-13.

Dörner, J., Dec, D., Peng, X., Horn, R., 2009a. Change of shrinkage behavior of an Andisol in southern Chile: Effects of land use and wetting/drying cycles. Soil and Tillage Reseacrh 106, 45-53.

Dörner, J., Dec, D., Peng, X., Horn, R., 2009b. Efecto del cambio de uso en la estabilidad de la estructura y la función de los poros de un Andisol (Typic Hapludand) del sur de Chile. Revista de la Ciencia del Suelo y Nutrición Vegetal 9, 190-209.

Dörner, J., Dec, D., Peng, X., Horn, R., 2010. Effect of land use change on the dynamic behaviour of structural properties of an Andisol in southern Chile under saturated and unsaturated hydraulic conditions. Geoderma 159, 189197.

Dörner, J., Dec, D., Zúñiga, F., Sandoval, P., Horn, R., 2011. Effect of land use change on Andosol's pore functions and their functional resilience after mechanical and hydraulic stresses. Soil and Tillage Research 115-116, 71-79.

Dörner, J., Dec, D., Feest, E., Vásquez, N., Díaz, M., 2012. Dynamics of soil structure and pore functions of a volcanic ash soil under tillage. Soil and Tillage Research 125, 5260.

Dörner, J., Dec, D., Zúñiga, F., Horn, R., López, I., Leiva, C., Cuevas, J., 2013a. Soil Changes in the Physical Quality of an Andosol under Different Management Intensities in Southern Chile, in: Krümmelbein, J., Horn, R., Pagliai, M. (Eds.). Soil Degradation, Advances in GeoEcology 42, GMBH Reiskirchen, Germany, pp. 262-281.

Dörner, J., Zúñiga, F., López, I., 2013b. Short-term effects of different pasture improvement treatments on the physical quality of an andisol. Journal of Soil Science and Plant Nutrition 2(13), 381-399.

Ellies, A., Ramírez, C., Mac Donald, R., 1993. Variación en la resistencia del suelo por efecto de su uso. Turrialba 43(1), 77-82.

Ellies, A., 1988. Mechanical consolidation in volcanic ash soils, in: Drescher, J., Horn, R., De Boodt, M. (Eds.), Impact of Water and External Forces on Soil Structure, Catena, Supplement 11, pp. 87-92.

Ellies, A., Horn, R., Smith, R., 2000. Effect of management of a volcanic ash soil on structural properties. International Agrophysics 14, 377-384.

Fujikawa, T., Miyazaki, T., 2005. Effect of bulk density and soil type on the gas diffusion coefficient in repacked and undisturbed soils. Soil Science 170, 892-901.

Gebhardt, S., Fleige, H., Horn, R., 2009. Effect of compaction on pore functions of soils in a Saalean moraine landscape in northern Germany. Journal of Plant Nutrition and Soil Science 172, 601-614.

Grossman, R.H., Brasher, B.R., Franzmeier, D.P., Walker, J.L., 1968. Linear extensibility as calculated from natural-clod bulk density measurements. Soil Science Society American Proccedings 32, 570-573.

Hartge, H., Horn, R., 2009. Die physikalische Untersuchung von Böden, vol. 4. Praxis Messmethoden Auswertung, Auflage, Schweizerbart, Stuttgart.

Hillel, D., 1998. Environmental Soil Physics. Academic Press, London.

Horn, R., Smucker, A., 2005. Structure formation and its consequences for gas and water transport in unsaturated arable and forest soils. Soil and Tillage Research 82, 5-14.

Horn, R., Fleige, H., 2009. Risk assessment of subsoil compaction for arable soils in Northwest Germany at farm scale. Soil and Tillage Research 102, 201-208.

Horn, R., Kutilek, M., 2009. The intensity-capacity conceptHow far is it possible to predict intensity values with capacity parameters. Soil and Tillage Research 103, 1-3.

Instituto Nacional de Estadísticas (INE). 2007. Informe estadísticas agropecuarias para el periodo 2001-2006 y primer semestre 2007http://www.ine.cl/canales/ 
chile_estadistico/estadisticas_agropecuarias/pdf/pecuarioprimersemestre2007_2.pdf (acesso 26.03.2016).

Ivelic-Sáez, J., Zúñiga, F., Valle, S., López, I., Dec, D., Dörner, J., 2015. Functional resistance and resilience of the pore system of an andisol exposed to different strategies of pasture improvement under sheep grazing. Journal of Soil Science and Plant Nutrition 15(3), 663-679.

Martínez, L.J., Zinc, J.A., 2004. Temporal variation of soil compaction and deterioration of soil quality in pasture areas of Colombian Amazonia. Soil and Tillage Research 75, 3-17.

Moldrup, P., Olesen, T., Komatsu, T., Schjønning, P., Rolston, D.E. 2001. Tortuosity, Diffusivity, and Permeability in the Soil Liquid and Gaseous Phases. Soil Science Society of American Journal 65, 613-623.

Oades, J.M., 1993. The role of biology in the formation, stabilization and degradation of soil structure. Geoderma 56(1-4), 377-400.

Osunbitan, J.A., Oyedele, D.J., Adekalu, K.O., 2005. Tillage effects on bulk density, hydraulic conductivity and strength of a loamy sands oil in Southwestern Nigeria. Soil and Tillage Research 82, 57-64.

Peng, X., Horn, R., Smucker, A., 2007. Pore shrinkage dependency of inorganic and organic soils on wetting and drying cycles. Soil Science Society of American Journal 71, 1095-1104.

Peng, X., Dörner, J., Zhao, Y., Horn, R., 2009. Shrinkage behaviour of transiently- and constantly-loaded soils and its consequences for soil moisture release. European Journal of Soil Science. 60, 681-694.

Peth, S., Horn, R., 2006. 11 Consequences of grazing on soil physical and mechanical properties in forest and tundra environments, in Ecological studies. Forbes, B.C., Bölter, M., Müller-Wille, L., Hukkinen, J., Müller, F., Gunslay, N.,
Konstatinov, Y., (Eds.), Vol. 184. Springer Verlag, Berlin Heilderberg, Germany, pp. 217-243.

Reszkowska, A., Krümmelbein, J., Gan, L., Peth, S., Horn, R., 2011. Influence of grazing on soil water and gas fluxes of two Inner Mongolian steppe ecosystems. Soil and Tillage Research 111, 180-189.

Richard, G., Cousin, I., Sillo, J.F., Bruand, A., Guerif, J., 2001. Effect of compaction on the porosity of a silty soil: influence on unsaturated hydraulic properties. European Journal of Soil Science 52, 49-58.

Sandoval, M., Dörner, J., Seguel, O., Cuevas, J., Rivera, D., 2012. Métodos de análisis físicos de suelos. Universidad de Concepción. Departamento de Suelos y Recursos Naturales, Número 5, Chillán, Chile.

Schwen, A., Bodner, G., Scholl, P., Buchan, G.D., Loiskandl, W., 2011. Temporal dynamics of soil hydraulic propierties and the water-conducting porosity under different tillage. Soil and Tillage Research 113, 89-98.

Schoenholtz, S.H., Van Miegroet, H., Burger, J.A., 2000. A review of chemical and physical properties as indicators of forest soil quality: challenges and opportunities. Forest Ecology and Management 138, 335-356.

Soil Survey Staff. 2006. Keys to Soil Taxonomy. $10^{\text {th }}$ ed. NRCS, Washington DC.

World Reference Base for Soil Resources (WRB). 2014. International soil classification system for naming soils and creating legends for soil maps. World Soil Resources Reports 106. Food and Agriculture Organization of the United Nations, Rome, Italy.

Zúñiga, F., Ivelic-Sáez, J., López, I., Huygens, D., Dörner, J., 2015. Temporal dynamics of the physical quality of an Andisol under a grazing system subjected to different pasture improvement strategies. Soil and Tillage Research 145, 233-241. 
